NIKHEF-2014-008

\title{
Trivalent graphs, volume conjectures and character varieties
}

\author{
Satoshi Nawata ${ }^{1}$, Ramadevi Pichai ${ }^{2}$, and Zodinmawia ${ }^{2}$
}

${ }^{1}$ NIKHEF theory group, Science Park 105, 1098 XG Amsterdam, The Netherlands

${ }^{2}$ Department of Physics, Indian Institute of Technology Bombay, India, 400076

\begin{abstract}
The generalized volume conjecture and the AJ conjecture (a.k.a. the quantum volume conjecture) are extended to $U_{q}\left(\mathfrak{s l}_{2}\right)$ colored quantum invariants of the theta and tetrahedron graph. The SL $(2, \mathbb{C})$ character variety of the fundamental group of the complement of a trivalent graph with $E$ edges in $S^{3}$ is a Lagrangian subvariety of the Hitchin moduli space over the Riemann surface of genus $g=E / 3+1$. For the theta and tetrahedron graph, we conjecture that the configuration of the character variety is locally determined by large color asymptotics of the quantum invariants of the trivalent graph in terms of complex Fenchel-Nielsen coordinates. Moreover, the $q$-holonomic difference equation of the quantum invariants provides the quantization of the character variety.
\end{abstract}

Mathematical Subject Classification (2010): 14D21, 53D30, 57M50, 81R50

Keywords: quantum invariants of trivalent graphs, generalized volume conjecture, AJ conjecture, character varieties

\section{Introduction and summary}

The volume conjecture provides a relation between colored Jones polynomials of a knot and the hyperbolic volume of the complement of the knot in $S^{3}$. After its formulation [19, 23], we have witnessed its extensions of various kinds which relate quantum invariants of knots and classical geometry of knot complements. In particular, the generalized volume conjecture [16] connects quantum invariants and character varieties of knot groups, and the AJ conjecture (a.k.a. the quantum volume conjecture) [12, 13, 16] gives the quantization of character varieties. In this letter, we will consider $U_{q}\left(\mathfrak{s l}_{2}\right)$ quantum invariants of simple planar graphs in the context of the generalized volume conjecture and the AJ conjecture.

A trivalent graph $\Gamma$ is a closed one-dimensional cell complex where three edges meet at each vertex. Therefore, the number $E$ of edges and that $V$ of vertices are related by $2 E=$ $3 V$. Colored $U_{q}\left(\mathfrak{s l}_{2}\right)$ quantum invariants $J_{n_{1}, \cdots, n_{E}}(\Gamma ; q)$ of a trivalent graph [5, 22, 28] can be obtained as follows. First, each edge of the graph carries a representation of $U_{q}\left(\mathfrak{s l}_{2}\right)$ as a color which is specified by an integer $n_{i} \in \mathbb{Z}$ corresponding to the spin- $n_{i} / 2$ representation of $U_{q}\left(\mathfrak{s l}_{2}\right)$. The colors $(a, b, c)$ on three edges meeting a trivalent vertex ${ }^{a} Y_{c}^{b}$ must obey the fusion rule, i.e. $|a-b| \leq c \leq a+b$. We also call a pair $(a, b, c)$ subject to the fusion rule an admissible set. Then, the quantum invariant of the colored trivalent graph can be obtained by summing 
over angular momentum states of $U_{q}\left(\mathfrak{s l}_{2}\right)$ Clebsch-Gordan $(q-\mathrm{CG})$ coefficients [20] associated to trivalent vertices. Specifically, this letter will deal with the first two simplest trivalent graphs: a theta graph (Figure 1) and a tetrahedron graph (Figure 2).

In fact, colored quantum invariants of trivalent graphs have been taken into account in terms of the volume conjecture [4, 6, 7, 24, 25, 28]. Namely, a suitable large color limit of quantum invariants of a trivalent graph can be related to the hyperbolic volume of the complement of the graph. Hence, our aim is to extend the generalized volume conjecture and the AJ conjecture (the quantum volume conjecture) for trivalent graphs.

For completeness, we will first briefly review the generalized volume conjecture and the AJ conjecture for colored Jones polynomials of knots. The generalized volume conjecture [16] amounts to the statement that in the double scaling limit, $n \rightarrow \infty, q=e^{\hbar} \rightarrow 1$ with $x=q^{n / 2}$ fixed, the asymptotic behavior of the colored Jones polynomials $J_{n}(K ; q)$, for any knot $K$ takes the form

$$
J_{n}(K ; q) \stackrel{\substack{n \rightarrow \infty \\ \hbar \rightarrow 0}}{\sim} \exp \left(\frac{1}{\hbar} \int \log y \frac{d x}{x}+\ldots\right),
$$

where the $y(x)$ essentially gives the zero locus of the classical $A$-polynomial $A(K ; x, y(x))=0$. The $A$-polynomial is also a knot invariant which describes the moduli space of $\operatorname{SL}(2, \mathbb{C})$ flat connections over the knot complement $S^{3} \backslash K$. This moduli space is also called as $\operatorname{SL}(2, \mathbb{C})$ character variety of the fundamental group of the knot complement $S^{3} \backslash K$. Recall that the moduli space $\mathscr{M}_{\text {flat }}\left(T^{2}, \mathrm{SL}(2, \mathbb{C})\right)$ of $\mathrm{SL}(2, \mathbb{C})$ flat connections on the boundary torus of the knot is a hyper-Kähler variety $\mathbb{C}^{\times} \times \mathbb{C}^{\times} / \mathbb{Z}_{2}$. The space $\mathbb{C}^{\times} \times \mathbb{C}^{\times}$is spanned by the holonomy eigenvalues of the $\operatorname{SL}(2, \mathbb{C})$ gauge connection along the meridian $x$ and the longitude $y$ of the torus, and $\mathbb{Z}_{2}$ is the Weyl group symmetry of the gauge group $\operatorname{SL}(2, \mathbb{C})$. The moduli space of $\mathrm{SL}(2, \mathbb{C})$ flat connections on the knot complement $S^{3} \backslash K$ is the Lagrangian subvariety of the moduli space $\mathscr{M}_{\text {flat }}\left(T^{2}, \mathrm{SL}(2, \mathbb{C})\right)$, with respect to the symplectic form $\omega=\frac{1}{\hbar} d \log x \wedge d \log y$, defined by the zero locus of the $A$-polynomial:

$$
\mathscr{M}_{\text {flat }}\left(S^{3} \backslash K, \mathrm{SL}(2, \mathbb{C})\right)=\left\{(x, y) \in \mathscr{M}_{\text {flat }}\left(T^{2}, \mathrm{SL}(2, \mathbb{C})\right) \mid A(K ; x, y)=0\right\} .
$$

Furthermore, the quantization of character varieties of knot groups $\pi_{1}\left(S^{3} \backslash K\right)$ can be obtained by considering the recursion relations of Jones polynomials, which is called the AJ conjecture [12, 13] or the quantum volume conjecture [16]. The recursion of the colored Jones polynomials of minimal order can be manipulated in the form

$$
\widehat{A}(K ; \hat{x}, \hat{y} ; q) J_{n}(K ; q)=0,
$$

where the operator $\hat{x}$ and $\hat{y}$ has the following action on the colored Jones polynomial:

$$
\hat{x} J_{n}(K ; q)=q^{n / 2} J_{n}(K ; q), \quad \hat{y} J_{n}(K ; q)=J_{n+1}(K ; q) .
$$

The quantum $A$-polynomial $\widehat{A}(K ; \hat{x}, \hat{y} ; q)$ reduces to the classical one at $q=1: \widehat{A}(K ; x, y ; q=$ $1)=A(K ; x, y)$.

It is natural to ask whether the generalized volume conjecture and the AJ conjecture are applicable for quantum invariants of a trivalent graph $\Gamma$ with $E$ edges colored by representations $n_{1}, \cdots, n_{E}$. Although one can consider knotted trivalent graphs in general, we restrict ourselves to the case of planar graphs, graphs which can be projected to the plane, for brevity in this paper. For a planar trivalent graph, the boundary of the tubular neighborhood of the graph $\Gamma$ is no longer a torus but a Riemann surface of genus $g \geq 2$. Actually, the genus is expressed by the number of edges via $g=E / 3+1$. This can be explained as follows. Let us project the trivalent graph $\Gamma$ on two-sphere $S^{2}$. This provides a cell decomposition of $S^{2}$ where the number $F$ of two-complex cells is indeed $F=g+1$. Since the Euler characteristics of $S^{2}$ is two, we have the relation

$$
V-E+F=2 .
$$


Using $2 E=3 V$ and $F=g+1$, we obtain $g=E / 3+1$. In fact, the moduli space $\mathscr{M}_{\text {flat }}\left(\Sigma_{g}, \mathrm{SL}(2, \mathbb{C})\right)$ of $\mathrm{SL}(2, \mathbb{C})$ flat connections on the boundary Riemann surface of genus $g$ is known as the Hitchin moduli space on $\Sigma_{g}$, which is a non-trivial hyper-Kähler variety of $\operatorname{dim}_{\mathbb{C}}=6 g-6=2 E$ [17]. Assigning a simple closed curve corresponding to each edge of the graph to the Riemann surface $\Sigma_{g}$, pants decomposition of $\Sigma_{g}$ is obtained. (See for example, Figure 1 and 2, As a result, the set of the $\operatorname{SL}(2, \mathbb{C})$ holonomy eigenvalue $x_{i}$ along a simple closed curve and its canonical conjugate "twist" variable $y_{i}$ becomes local Darboux coordinates of the Hitchin moduli space, so-called complex Fenchel-Nielsen coordinates [15, 9, 10]. Since the moduli space $\mathscr{M}_{\text {flat }}\left(S^{3} \backslash \Gamma, \mathrm{SL}(2, \mathbb{C})\right)$ of $\mathrm{SL}(2, \mathbb{C})$ flat connections on the complement of the trivalent graph $\Gamma$ in $S^{3}$ is a Lagrangian subvariety of $\mathscr{M}_{\text {flat }}\left(\Sigma_{g}, \mathrm{SL}(2, \mathbb{C})\right)$, the configuration of the moduli space $\mathscr{M}_{\text {flat }}\left(S^{3} \backslash \Gamma, \mathrm{SL}(2, \mathbb{C})\right) \subset \mathscr{M}_{\text {flat }}\left(\Sigma_{g}, \mathrm{SL}(2, \mathbb{C})\right)$ can be locally specified by complex Fenchel-Nielsen coordinates. Now, one can ask a question whether there is a relation between colored quantum invariants $J_{n_{1}, \cdots, n_{E}}(\Gamma ; q)$ of a graph and the the configuration of the moduli space $\mathscr{M}_{\text {flat }}\left(S^{3} \backslash \Gamma, \mathrm{SL}(2, \mathbb{C})\right)$.

In what follows, we will make a modest step to answer to this question for the theta and tetrahedron graph. Therefore, let $\Gamma$ be either the theta or tetrahedron graph and $J_{n_{1}, \cdots, n_{E}}(\Gamma ; q)$ be its colored quantum invariants. In the double scaling limit $q=e^{\hbar} \rightarrow 1$ and $n_{i} \rightarrow \infty$ with $q^{n_{i} / 2}=x_{i}$ fixed $(i=1, \cdots, E)$, we conjecture that the large color asymptotics of quantum invariants of $\Gamma$ exhibits the form

$$
J_{n_{1}, \cdots, n_{E}}(\Gamma ; q) \stackrel{\substack{n_{i} \rightarrow \infty \\ \hbar \rightarrow 0}}{\sim} e^{\frac{1}{\hbar} W\left(\Gamma ;\left\{x_{i}\right\}\right)}=\exp \left(\frac{1}{\hbar} \int \sum_{i} \log y_{i} \frac{d x_{i}}{x_{i}}+\ldots\right) .
$$

where the integral is performed over the moduli space $\mathscr{M}_{\text {flat }}\left(S^{3} \backslash \Gamma, \mathrm{SL}(2, \mathbb{C})\right)$ determined by $3 g-3=E$ set of equations

$$
A_{j}\left(\Gamma ; y_{j}, x_{1}, \ldots, x_{E}\right)=0, \quad j=1, \cdots, E .
$$

Note that $\left\{x_{i}, y_{i}\right\}_{i=1, \cdots, E}$ are complex Fenchel-Nielsen coordinates of the Hitchin moduli space. This set of equations can be obtained by solving the equations

$$
y_{j}=\exp \left(x_{j} \frac{\partial W\left(\Gamma ;\left\{x_{i}\right\}\right)}{\partial x_{j}}\right) .
$$

Furthermore, the moduli space $\mathscr{M}_{\text {flat }}\left(S^{3} \backslash \Gamma, \mathrm{SL}(2, \mathbb{C})\right)$ defined by $(7)$ is the Lagrangian subvariety of $\operatorname{dim}_{\mathbb{C}}=3 g-3=E$ in the moduli space $\mathscr{M}_{\text {flat }}\left(\Sigma_{g}, \mathrm{SL}(2, \mathbb{C})\right)$ with respect to the symplectic form $\omega=\frac{1}{\hbar} \sum_{i=1}^{E} d \log x_{i} \wedge d \log y_{i}$.

It is straightforward to formulate the AJ conjecture for the theta and tetrahedron graph. Similar to the operator actions $\hat{x}$ and $\hat{y}$ in (4), let us define the acton of operators $\hat{x}_{j}$ and $\hat{y}_{j}$ $(j=1, \ldots, E)$ on the graph invariant as follows:

$$
\begin{aligned}
& \hat{x}_{j} J_{n_{1}, \cdots, n_{E}}(\Gamma ; q)=q^{n_{j} / 2} J_{n_{1}, \cdots, n_{E}}(\Gamma ; q) \\
& \hat{y}_{j} J_{n_{1}, \cdots, n_{E}}(\Gamma ; q)=J_{n_{1}, \cdots, n_{j}+2, \cdots, n_{E}}(\Gamma ; q),
\end{aligned}
$$

We should stress out that the fusion rule allows to increase the rank of a representation by two boxes in terms of Young tableaux unlike the case of knot invariants. Then, a recursion relation of minimal order with respect to one of colors

$$
b_{k}^{(j)}\left(\hat{x}_{1}, \ldots, \hat{x}_{E}, q\right) J_{n_{1}, \cdots, n_{j}+2 k, \cdots, n_{E}}(\Gamma ; q)+\cdots+b_{0}^{(j)}\left(\hat{x}_{1}, \ldots, \hat{x}_{E}, q\right) J_{n_{1}, \cdots, n_{j}, \cdots, n_{E}}(\Gamma ; q)=0,
$$

can be written as

$$
\widehat{A}_{j}\left(\Gamma ; \hat{y}_{j}, \hat{x}_{1}, \ldots, \hat{x}_{E} ; q\right) J_{n_{1}, \cdots, n_{E}}(\Gamma ; q)=0
$$

where

$$
\widehat{A}_{j}\left(\Gamma ; \hat{y}_{j}, \hat{x}_{1}, \ldots, \hat{x}_{E} ; q\right)=\sum_{\ell=0}^{k} b_{\ell}^{(j)}\left(\hat{x}_{1}, \ldots, \hat{x}_{E}, q\right) \hat{y}_{j}^{\ell}
$$


The set of the operators $\widehat{A}_{j}\left(\Gamma ; \hat{y}_{j},\left\{\hat{x}_{i}\right\} ; q\right)(i=1, \ldots, E)$ provides the quantization of the character variety $\mathscr{M}_{\text {flat }}\left(S^{3} \backslash \Gamma, \mathrm{SL}(2, \mathbb{C})\right)$ defined by $(7)$ in the sense that

$$
\widehat{A}_{j}\left(\Gamma ; y_{j},\left\{x_{i}\right\} ; q=1\right)=A_{j}\left(\Gamma ; y_{j},\left\{x_{i}\right\}\right) \text {. }
$$

In the following sections, we will demonstrate explicit calculations of $A_{j}\left(\Gamma ; y_{j},\left\{x_{i}\right\}\right)$ for the theta and tetrahedron graph.

\section{Theta graph}

A theta graph is the simplest trivalent graph with three edges $E=3$ and two trivalent vertices $V=2$ as shown in Figure 1. The colored quantum invariant $J_{a, b, c}(\Theta ; q)(a, b, c \in \mathbb{Z})$ of the theta graph can be written as a state sum involving product of two Clebsch-Gordan coefficients [5]. In [22], the invariants of the theta graph is written in the succinct form by using the Wenzl's recursion formula for Jones idempotents of the Temperly-Lieb algebra

$$
J_{a, b, c}(\Theta ; q)=(-1)^{(a+b+c) / 2} \frac{[(a+b+c) / 2+1] ![(-a+b+c) / 2] ![(a-b+c) / 2] ![(a+b-c) / 2] !}{[a] ![b] ![c] !}
$$

The numbers in square brackets are known as $q$-number defined by

$$
[n]=\frac{q^{n / 2}-q^{-n / 2}}{q^{1 / 2}-q^{-1 / 2}},
$$

where $q \rightarrow 1$ gives the number $n$. Similarly, a $q$-factorial is given by $[n] !=[n][n-1] \ldots[3][2][1]$.
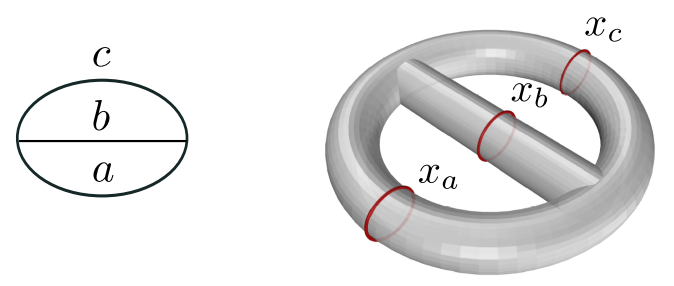

Figure 1: Theta graph and its tubular neighborhood.

\subsection{Classical character variety}

As shown in Figure 1, the tubular neighborhood of the theta graph is a genus-two handlebody. Hence, the moduli space $\mathscr{M}_{\text {flat }}\left(\Sigma_{g=2}, \mathrm{SL}(2, \mathbb{C})\right)$ of $\mathrm{SL}(2, \mathbb{C})$ flat connections on the boundary Riemann surface $\Sigma_{g=2}$ of genus-two is a hyper-Kähler variety of $\operatorname{dim}_{\mathbb{C}}=6$ [17] whose local coordinates are spanned by the holonomy eigenvalues $\left(x_{a}, x_{b}, x_{c}\right)$ and twist coordinates $\left(y_{a}, y_{b}, y_{c}\right)$. The moduli space $\mathscr{M}_{\text {flat }}\left(S^{3} \backslash \Theta, \operatorname{SL}(2, \mathbb{C})\right)$ of $\operatorname{SL}(2, \mathbb{C})$ flat connections on the complement $S^{3} \backslash \Theta$ is a Lagrangian subvariety of the moduli space $\mathscr{M}_{\text {flat }}\left(\Sigma_{g=2}, \mathrm{SL}(2, \mathbb{C})\right)$ with respect to the symplectic form $\omega=\frac{1}{\hbar} \sum_{i} d \log x_{i} \wedge d \log y_{i}$, which is described by the zero loci of the set of algebraic equations $A_{j}\left(\Theta ; y_{j},\left\{x_{i}\right\}\right)=0(j=a, b, c)$

$$
\begin{aligned}
& \mathscr{M}_{\text {flat }}\left(S^{3} \backslash \Theta, \operatorname{SL}(2, \mathbb{C})\right) \\
= & \left\{\left(x_{a}, x_{b}, x_{c}, y_{a}, y_{b}, y_{c}\right) \in \mathscr{M}_{\text {flat }}\left(\Sigma_{g=2}, \operatorname{SL}(2, \mathbb{C})\right) \mid A_{j}\left(\Theta ; y_{j},\left\{x_{i}\right\}\right)=0 \quad(j=a, b, c)\right\} .
\end{aligned}
$$

The generalized volume conjecture states that the set of equations can be obtained by taking large color limit of the $U_{q}\left(\mathfrak{s l}_{2}\right)$ quantum invariants of the theta graph. In order to explicitly see 
the large color behavior, we use the asymptotic form of $[n]$ ! in the limit of $q=e^{\hbar} \rightarrow 1, n \rightarrow \infty$ with $q^{n}=x_{n}$ fixed

$$
[n] ! \stackrel{\hbar \rightarrow 0, n \rightarrow \infty}{\longrightarrow} e^{\frac{1}{\hbar} g\left(x_{n}\right)}, \quad \text { where } \quad g\left(x_{n}\right)=-\frac{\left(\log x_{n}\right)^{2}}{4}-\operatorname{Li}_{2}\left(x_{n}\right) .
$$

Thus, in the limit

$$
\hbar \rightarrow 0, \quad(a, b, c) \rightarrow \infty, \quad x_{a}=q^{a / 2}=\text { fixed }, \quad x_{b}=q^{b / 2}=\text { fixed }, \quad x_{c}=q^{c / 2}=\text { fixed },
$$

the quantum invariant of the theta graph (14) takes the form

$$
J_{a, b, c}(\Theta) \sim e^{\frac{1}{\hbar} W\left(\Theta ; x_{a}, x_{b}, x_{c}\right)},
$$

where the Neumann-Zagier potential is written by

$$
\begin{aligned}
W\left(\Theta ; x_{a}, x_{b}, x_{c}\right)= & \pi i \log \left(x_{a} x_{b} x_{c}\right)+g\left(x_{a} x_{b} x_{c}\right)+g\left(x_{a}^{-1} x_{b} x_{c}\right)+g\left(x_{a} x_{b}^{-1} x_{c}\right)+g\left(x_{a} x_{b} x_{c}^{-1}\right) \\
& -g\left(x_{a}^{2}\right)-g\left(x_{b}^{2}\right)-g\left(x_{c}^{2}\right)
\end{aligned}
$$

The set of equations which determines the moduli space $\mathscr{M}_{\text {flat }}\left(S^{3} \backslash \Theta, \mathrm{SL}(2, \mathbb{C})\right)$ can be obtained from

$$
y_{j}=\exp \left(x_{j} \frac{\partial W\left(\Theta ;\left\{x_{i}\right\}\right)}{\partial x_{j}}\right),
$$

Hence, plugging the Neumann-Zagier potential $19 p$ into the equations, we conjecture that the moduli space $\mathscr{M}_{\text {flat }}\left(S^{3} \backslash \Theta, \operatorname{SL}(2, \mathbb{C})\right)$ is determined by the zero loci of the following equations

$$
\begin{aligned}
& A_{a}\left(\Theta ; y_{a},\left\{x_{i}\right\}\right)=x_{a}\left(x_{a} x_{b}-x_{c}\right)\left(x_{a} x_{c}-x_{b}\right)\left(1-x_{a} x_{b} x_{c}\right)+x_{b} x_{c}\left(1-x_{a}^{2}\right)^{2}\left(x_{a}-x_{b} x_{c}\right) y_{a}, \\
& A_{b}\left(\Theta ; y_{b},\left\{x_{i}\right\}\right)=x_{b}\left(x_{a} x_{b}-x_{c}\right)\left(x_{b} x_{c}-x_{a}\right)\left(1-x_{a} x_{b} x_{c}\right)+x_{a} x_{c}\left(1-x_{b}^{2}\right)^{2}\left(x_{b}-x_{a} x_{c}\right) y_{b}, \\
& A_{c}\left(\Theta ; y_{c},\left\{x_{i}\right\}\right)=x_{c}\left(x_{a} x_{c}-x_{b}\right)\left(x_{b} x_{c}-x_{a}\right)\left(1-x_{a} x_{b} x_{c}\right)+x_{a} x_{b}\left(1-x_{c}^{2}\right)^{2}\left(x_{c}-x_{a} x_{b}\right) y_{c},
\end{aligned}
$$

in the moduli space $\mathscr{M}_{\text {flat }}\left(\Sigma_{g=2}, \mathrm{SL}(2, \mathbb{C})\right)$. In fact, we have checked that the subvariety defined by the zero loci of the set of the equations (21) satisfy the Lagrangian condition. Namely, the symplectic form $\omega=\frac{1}{\hbar} \sum_{i} d \log x_{i} \wedge d \log y_{i}$ vanishes on the subvariety. We conjecture that the set (21) of the equations locally determines the moduli space $\mathscr{M}_{\text {flat }}\left(S^{3} \backslash \Theta, \operatorname{SL}(2, \mathbb{C})\right) \subset$ $\mathscr{M}_{\text {flat }}\left(\Sigma_{g=2}, \mathrm{SL}(2, \mathbb{C})\right)$. It is desirable to obtain the $\mathrm{SL}(2, \mathbb{C})$ character variety of the fundamental group $\pi_{1}\left(S^{3} \backslash \Theta\right)$ in terms of the complex Fenchel-Nielsen coordinates $\left\{x_{i}, y_{i}\right\}$ by the other means.

\subsection{Quantum character variety}

In order to quantize the classical character varieties $A_{i}\left(\left\{x_{i}\right\}, y_{i}\right)$, we introduce operators $\hat{x}_{i}$ and $\hat{y}_{i}$, whose actions on $J_{a, b, c}(\Theta ; q)$ are as follows:

$$
\begin{aligned}
& \hat{x}_{a} J_{a, b, c}(\Theta, q)=q^{a / 2} J_{a, b, c}(\Theta, q) \\
& \hat{y}_{a} J_{a, b, c}(\Theta, q)=J_{a+2, b, c}(\Theta, q)
\end{aligned}
$$

From the following recursion relation for $J_{a, b, c}(\Theta, q)$

$$
J_{a+2, b, c}(\Theta, q)=-\frac{[(a+b+c) / 2+2][(a-b+c) / 2+1][(a+b-c) / 2+1]}{[(-a+b+c) / 2][a+1][a+2]} J_{a, b, c}(\Theta, q),
$$

we can deduce the form for the quantum character variety as

$$
\begin{aligned}
\widehat{A}_{a}\left(\Theta ; \hat{y}_{a},\left\{\hat{x}_{i}\right\}\right)= & \hat{x}_{a}\left(q \hat{x}_{a} \hat{x}_{b}-\hat{x}_{c}\right)\left(q \hat{x}_{a} \hat{x}_{c}-\hat{x}_{b}\right)\left(1-q^{2} \hat{x}_{a} \hat{x}_{b} \hat{x}_{c}\right) \\
& +q^{-3 / 2} \hat{x}_{b} \hat{x}_{c}\left(1-\hat{x}_{a}^{2}\right)\left(q-\hat{x}_{a}^{2}\right)\left(\hat{x}_{a}-q \hat{x}_{b} \hat{x}_{c}\right) \hat{y}_{a},
\end{aligned}
$$




$$
\begin{aligned}
\widehat{A}_{b}\left(\Theta ; \hat{y}_{b},\left\{\hat{x}_{i}\right\}\right)= & \hat{x}_{b}\left(q \hat{x}_{a} \hat{x}_{b}-\hat{x}_{c}\right)\left(q \hat{x}_{b} \hat{x}_{c}-\hat{x}_{a}\right)\left(1-q^{2} \hat{x}_{a} \hat{x}_{b} \hat{x}_{c}\right) \\
& +q^{-3 / 2} \hat{x}_{a} \hat{x}_{c}\left(1-\hat{x}_{b}^{2}\right)\left(q-\hat{x}_{b}^{2}\right)\left(\hat{x}_{b}-q \hat{x}_{a} \hat{x}_{c}\right) \hat{y}_{b}, \\
\widehat{A}_{c}\left(\Theta ; \hat{y}_{c},\left\{\hat{x}_{i}\right\}\right)= & \hat{x}_{c}\left(q \hat{x}_{a} \hat{x}_{c}-\hat{x}_{b}\right)\left(q \hat{x}_{b} \hat{x}_{c}-\hat{x}_{a}\right)\left(1-q^{2} \hat{x}_{a} \hat{x}_{b} \hat{x}_{c}\right) \\
& +q^{-3 / 2}\left(\hat{x}_{a} \hat{x}_{b} 1-\hat{x}_{c}^{2}\right)\left(q-\hat{x}_{c}^{2}\right)\left(\hat{x}_{c}-q \hat{x}_{a} \hat{x}_{b}\right) \hat{y}_{c} .
\end{aligned}
$$

Our proposal of the quantization of the character variety for the theta graph is evident from the equality of $q \rightarrow 1$ limit of 24 with the classical character varieties (21).

\section{Tetrahedron graph}

The colored quantum invariants $J_{j_{1}, \ldots j_{4}, j_{12}, j_{23}}(\bigotimes ; q)$ for the tetrahedron graph are know as the quantum $6 j$-symbols for $U_{q}\left(\mathfrak{s l}_{2}\right)$ [21], up to a certain factor, which are written as state sum of product of four $q-\mathrm{CG}$ coefficients. In this paper, we adopt the notation used in [22]
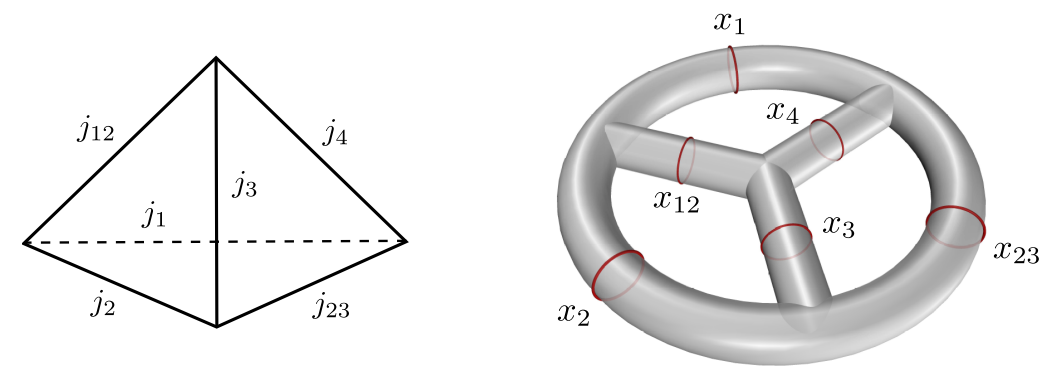

Figure 2: Tetrahedron graph and its tubular neighborhood.

$$
\begin{aligned}
\left\langle\begin{array}{lll}
j_{1} & j_{2} & j_{12} \\
j_{3} & j_{4} & j_{23}
\end{array}\right\rangle= & \frac{\Delta\left(j_{1}, j_{2}, j_{12}\right) \Delta\left(j_{3}, j_{4}, j_{12}\right) \Delta\left(j_{1}, j_{4}, j_{23}\right) \Delta\left(j_{2}, j_{3}, j_{23}\right)}{\left[j_{1}\right] !\left[j_{2}\right] !\left[j_{3}\right] !\left[j_{4}\right] !\left[j_{12}\right] !\left[j_{23}\right] !} \\
& \times \sum_{m \geq 0}(-)^{m}[m+1] !\left\{\left[m-\frac{1}{2}\left(j_{1}-j_{2}-j_{12}\right)\right] !\right. \\
& \times\left[m-\frac{1}{2}\left(j_{3}-j_{4}-j_{12}\right)\right] !\left[m-\frac{1}{2}\left(j_{1}-j_{4}-j_{23}\right)\right] ! \\
& \times\left[m-\frac{1}{2}\left(j_{2}-j_{3}-j_{23}\right)\right] !\left[\frac{1}{2}\left(j_{1}+j_{2}+j_{3}+j_{4}\right)-m\right] ! \\
& \left.\times\left[\frac{1}{2}\left(j_{1}+j_{3}+j_{12}+j_{23}\right)-m\right] !\left[\frac{1}{2}\left(j_{2}+j_{4}+j_{12}+j_{23}\right)-m\right] !\right\}^{-1}
\end{aligned}
$$

where we define

$$
\Delta(a, b, c)=[(-a+b+c) / 2] ![(a-b+c) / 2] ![(a+b-c) / 2] ! .
$$

Note that $j_{i} \in \mathbb{Z}$ represent the spin- $j_{i} / 2$ representations of $U_{q}\left(\mathfrak{s l}_{2}\right)$ assigned to the six edges (Figure 2). If a singlet representation is placed on an edge of the tetrahedron, the tetrahedron invariant reduces to the quantum invariant (14) of the theta graph. It is well-known that the tetrahedron invariant exhibits the following symmetry properties:

$$
\left\langle\begin{array}{lll}
j_{1} & j_{2} & j_{12} \\
j_{3} & j_{4} & j_{23}
\end{array}\right\rangle=\left\langle\begin{array}{lll}
j_{2} & j_{1} & j_{12} \\
j_{4} & j_{3} & j_{23}
\end{array}\right\rangle=\left\langle\begin{array}{lll}
j_{12} & j_{2} & j_{1} \\
j_{23} & j_{4} & j_{3}
\end{array}\right\rangle=\left\langle\begin{array}{lll}
j_{3} & j_{2} & j_{23} \\
j_{1} & j_{4} & j_{12}
\end{array}\right\rangle=\left\langle\begin{array}{lll}
j_{3} & j_{4} & j_{12} \\
j_{1} & j_{2} & j_{23}
\end{array}\right\rangle .
$$

In what follows, we shall obtain the classical and quantum character varieties of the complement of the tetrahedron graph in $S^{3}$. To this end, we will ignore the factor in front of the summation in (25) since the effect of the factor can be absorbed into the redefinition of the variables $y_{i}$. It turns out 21] that the summation part can be written in terms of the basic hypergeometric series

$$
\left\langle\begin{array}{lll}
j_{1} & j_{2} & j_{12} \\
j_{3} & j_{4} & j_{23}
\end{array}\right\rangle^{\prime}
$$




$$
\begin{aligned}
= & \frac{(-)^{\left(j_{1}+j_{2}+j_{3}+j_{4}\right) / 2}\left[\frac{1}{2}\left(j_{1}+j_{2}+j_{3}+j_{4}\right)+1\right] !}{\left[\frac{1}{2}\left(j_{1}+j_{2}-j_{12}\right)\right] !\left[\frac{1}{2}\left(j_{1}+j_{4}-j_{23}\right)\right] !\left[\frac{1}{2}\left(j_{3}+j_{4}-j_{12}\right)\right] !\left[\frac{1}{2}\left(j_{2}+j_{3}-j_{12}\right)\right] !\left[\frac{1}{2}\left(j_{12}+j_{23}-j_{2}-j_{4}\right)\right] !\left[\frac{1}{2}\left(j_{12}+j_{23}-j_{1}-j_{2}\right)\right] !} \\
& { }_{4} \varphi_{3}\left(\begin{array}{c}
q^{\frac{1}{2}\left(-j_{1}-j_{2}+j_{12}\right)}, q^{\frac{1}{2}\left(-j_{1}-j_{4}+j_{23}\right)}, q^{\frac{1}{2}\left(-j_{3}-j_{4}+j_{12}\right)}, q^{\frac{1}{2}\left(-j_{2}-j_{3}+j_{12}\right)} \\
q^{-\frac{1}{2}\left(j_{1}+j_{2}+j_{3}+j_{4}\right)-1}, q^{\frac{1}{2}\left(j_{12}+j_{23}-j_{2}-j_{4}\right)+1}, q^{\frac{1}{2}\left(j_{12}+j_{23}-j_{1}-j_{2}\right)+1}
\end{array} \mid q, q\right)
\end{aligned}
$$

\subsection{Classical character variety}

It is easy to see that the tubular neighborhood of the tetrahedron graph is a genus-3 handlebody. Hence, as Figure 2 depicted, the moduli space $\mathscr{M}_{\text {flat }}\left(\Sigma_{g=3}, \mathrm{SL}(2, \mathbb{C})\right)$ of $\mathrm{SL}(2, \mathbb{C})$ flat connections on the boundary Riemann surface $\Sigma_{g=3}$ will be a 12-dimensional hyper-Kähler variety [17] whose coordinates are spanned by the holonomy eigenvalues $x_{i}(i \in\{1, \cdots, 4,12,23\})$ and their conjugate variables $y_{i}(i \in\{1, \cdots, 4,12,23\})$. The classical character variety $\mathscr{M}_{\text {flat }}\left(S^{3} \backslash \bigotimes, \operatorname{SL}(2, \mathbb{C})\right)$ of the complement of the tetrahedron graph is determined by a set of six Laurent polynomials

$$
A_{k}\left(\bigotimes ; y_{k},\left\{x_{i}\right\}\right)=0, \quad k \in\{1, \cdots, 4,12,23\},
$$

which cuts out the Lagrangian subvariety of the moduli space $\mathscr{M}_{\text {flat }}\left(\Sigma_{g=3}, \mathrm{SL}(2, \mathbb{C})\right)$. To see the explicit form of 29 via the generalized volume conjecture, we will take the limit

$$
q=e^{\hbar} \rightarrow 1, \quad j_{i} \rightarrow \infty, \quad x_{i}=q^{j_{i} / 2}=\text { fixed }, \quad \mathrm{i} \in\{1, \cdots, 4,12,23\}
$$

of the colored quantum invariants of the tetrahedron graph. In this limit, the summation over $m$ in 28 is approximated an integral over a variable $z=q^{m}$

$$
\left\langle\begin{array}{lll}
j_{1} & j_{2} & j_{12} \\
j_{3} & j_{4} & j_{23}
\end{array}\right\rangle^{\prime} \sim \int e^{\left.\frac{1}{\hbar} \widetilde{\mathcal{W}}(\otimes) ;\left\{x_{i}\right\} ; z\right)} d z
$$

where the Neumann-Zagier potential can be read off from 116 as

$$
\begin{aligned}
\widetilde{\mathcal{W}}\left(\bigotimes ;\left\{x_{i}\right\}, z\right)= & \pi i \log (z)+g(z) \\
& -g\left(\frac{z}{x_{j_{1}} x_{j_{2}} x_{j_{12} 2}}\right)-g\left(\frac{z}{x_{j_{3}} x_{j_{4}} x_{j_{12}}}\right)-g\left(\frac{z}{x_{j_{1}} x_{j_{4}} x_{j_{23}}}\right)-g\left(\frac{z}{x_{j_{2}} x_{j_{3}} x_{j_{23}}}\right) \\
& -g\left(\frac{x_{j_{1}} x_{j_{2}} x_{j_{3}} x_{j_{4}}}{z}\right)-g\left(\frac{x_{j_{1}} x_{j_{12}} x_{j_{3}} x_{j_{23}}}{z}\right)-g\left(\frac{x_{j_{2}} x_{j_{12}} x_{j_{4}} x_{j_{23}}}{z}\right)
\end{aligned}
$$

The leading contribution of the asymptotics comes from the saddle point $z=z_{0}$

$$
\left.\frac{\partial \widetilde{\mathcal{W}}\left(\bigotimes ;\left\{x_{i}\right\}, z\right)}{\partial z}\right|_{z=z_{0}}=0
$$

Therefore, the zero locus of the classical character variety is determined by

$$
y_{k}=\exp \left(x_{k} \frac{\partial \widetilde{\mathcal{W}}\left(\bigotimes ;\left\{x_{i}\right\}, z_{0}\right)}{\partial x_{k}}\right) .
$$

Substituting the Neumann-Zagier potential $(32)$ into the above equations for $k=1$, we have

$$
\begin{aligned}
1 & =\frac{\left(z_{0}-1\right)\left(z_{0}-x_{1} x_{2} x_{3} x_{4}\right)\left(z_{0}-x_{1} x_{3} x_{12} x_{23}\right)\left(z_{0}-x_{2} x_{4} x_{12} x_{23}\right)}{\left(z_{0}-x_{1} x_{2} x_{12}\right)\left(z_{0}-x_{1} x_{4} x_{23}\right)\left(z_{0}-x_{2} x_{3} x_{23}\right)\left(z_{0}-x_{3} x_{4} x_{12}\right)} \\
y_{1} & =\frac{x_{3}\left(z_{0}-x_{1} x_{4} x_{23}\right)\left(z_{0}-x_{1} x_{2} x_{12}\right)}{\left(z_{0}-x_{1} x_{2} x_{3} x_{4}\right)\left(z_{0}-x_{1} x_{3} x_{12} x_{23}\right)} .
\end{aligned}
$$

Eliminating $z_{0}$ from these two equations, we obtain an explicit expression

$$
\begin{aligned}
A_{1}\left(\bigotimes ; y_{1},\left\{x_{i}\right\}\right)= & x_{3}\left(x_{1} x_{4}-x_{23}\right)\left(x_{4}-x_{1} x_{23}\right)\left(x_{1} x_{2}-x_{12}\right)\left(x_{2}-x_{1} x_{12}\right) y_{1}^{2} \\
& -\left[x_{4} x_{12}\left(1-x_{1}^{2}\right)^{2}\left(x_{3}-x_{2} x_{23}\right)\left(1-x_{3} x_{2} x_{23}\right)\right. \\
& +x_{3}\left(x_{23}-x_{1} x_{4}\right)\left(x_{1}-x_{4} x_{23}\right)\left(x_{1}-x_{2} x_{12}\right)\left(x_{2}-x_{1} x_{12}\right)
\end{aligned}
$$




$$
\begin{aligned}
& \left.+x_{3}\left(x_{4}-x_{1} x_{23}\right)\left(1-x_{1} x_{4} x_{23}\right)\left(x_{12}-x_{1} x_{2}\right)\left(1-x_{1} x_{2} x_{12}\right)\right] y_{1} \\
& +x_{3}\left(x_{1}-x_{4} x_{23}\right)\left(x_{1}-x_{2} x_{12}\right)\left(1-x_{1} x_{4} x_{23}\right)\left(1-x_{1} x_{2} x_{12}\right) .
\end{aligned}
$$

From this expression, we can find the other formulas by using the symmetry property (27),

$$
\begin{aligned}
A_{2}\left(\bigotimes ; y_{2},\left\{x_{i}\right\}\right) & =A_{2}\left(\bigotimes ; y_{1} \rightarrow y_{2}, x_{2} \leftrightarrow x_{1}, x_{3} \leftrightarrow x_{4}\right), \\
A_{3}\left(\bigotimes ; y_{3},\left\{x_{i}\right\}\right) & =A_{1}\left(\bigotimes ; y_{1} \rightarrow y_{3}, x_{3} \leftrightarrow x_{1}, x_{23} \leftrightarrow x_{12}\right), \\
A_{4}\left(\bigotimes ; y_{4},\left\{x_{i}\right\}\right) & =A_{1}\left(\bigotimes ; y_{1} \rightarrow y_{4}, x_{3} \leftrightarrow x_{2}, x_{1} \leftrightarrow x_{4}\right), \\
A_{12}\left(\bigotimes ; y_{12},\left\{x_{i}\right\}\right) & =A_{1}\left(\bigotimes ; y_{1} \rightarrow y_{12}, x_{3} \leftrightarrow x_{23}, x_{1} \leftrightarrow x_{12}\right), \\
A_{23}\left(\bigotimes ; y_{23},\left\{x_{i}\right\}\right) & =A_{1}\left(\bigotimes ; y_{1} \rightarrow y_{23}, x_{3} \leftrightarrow x_{12}, x_{1} \leftrightarrow x_{23}\right) .
\end{aligned}
$$

Thus, we conjecture that the zero locus of these six polynomial equations represents the classical character variety of the tetrahedron graph in the moduli space $\mathscr{M}_{\text {flat }}\left(\Sigma_{g=3}, \mathrm{SL}(2, \mathbb{C})\right)$. Indeed, we have verified that it is subject to the Lagrangian condition by showing the symplectic form $\omega=\frac{i}{\hbar} \sum_{i} d \log x_{i} \wedge d \log y_{i}=0$ on the subvariety.

\subsection{Quantum character variety}

As we have seen, the colored quantum invariants of the tetrahedron graph can be written in terms of a balanced basic hypergeometric series ${ }_{4} \varphi_{3}$. Moreover, it is a certain orthogonal polynomial called an Askey-Wilson polynomial [2]. Thus, we can obtain the recursion relation [2, 18] which is explicitly written as

$$
\alpha\left\langle\begin{array}{ccc}
j_{1}+2 & j_{2} & j_{12} \\
j_{3} & j_{4} & j_{23}
\end{array}\right\rangle^{\prime}-\beta\left\langle\begin{array}{ccc}
j_{1} & j_{2} & j_{12} \\
j_{3} & j_{4} & j_{23}
\end{array}\right\rangle^{\prime}+\gamma\left\langle\begin{array}{ccc}
j_{1}-2 & j_{2} & j_{12} \\
j_{3} & j_{4} & j_{23}
\end{array}\right\rangle^{\prime}=0,
$$

where

$$
\begin{aligned}
\alpha= & {\left[j_{1}\right]\left[\left(j_{1}+j_{4}-j_{23}\right) / 2+1\right]\left[\left(j_{1}+j_{23}-j_{4}\right) / 2+1\right]\left[\left(j_{1}+j_{2}-j_{12}\right) / 2+1\right]\left[\left(j_{1}+j_{12}-j_{2}\right) / 2+1\right] } \\
\beta= & {\left[j_{1}+2\right]\left[\left(j_{1}+j_{23}-j_{4}\right) / 2\right]\left[\left(j_{1}+j_{4}+j_{23}\right) / 2+1\right]\left[\left(j_{1}+j_{2}-j_{12}\right) / 2\right]\left[\left(j_{1}+j_{2}+j_{12}\right) / 2+1\right] } \\
& +\left[j_{1}\right]\left[\left(j_{1}+j_{4}-j_{23}\right) / 2+1\right]\left[\left(j_{23}+j_{4}-j_{1}\right) / 2\right]\left[\left(j_{2}+j_{12}-j_{1}\right) / 2\right]\left[\left(j_{1}+j_{12}-j_{2}\right) / 2+1\right] \\
& -\left[j_{1}\right]\left[j_{1}+1\right]\left[j_{1}+2\right]\left[\left(j_{3}+j_{2}+j_{23}\right) / 2+1\right]\left[\left(j_{2}+j_{23}-j_{3}\right) / 2\right] \\
\gamma= & {\left[j_{1}+2\right]\left[\left(j_{1}+j_{4}+j_{23}\right) / 2+1\right]\left[\left(j_{23}+j_{4}-j_{1}\right) / 2+1\right]\left[\left(j_{2}+j_{12}+j_{1}\right) / 2+1\right] } \\
& \times\left[\left(j_{2}+j_{12}-j_{1}\right) / 2+1\right] .
\end{aligned}
$$

Using the following action of the quantum operator $\hat{x}_{i}$ and the conjugate operator $\hat{y}_{1}$ for the tetrahedron invariant

$$
\begin{aligned}
& \hat{x}_{i}\left\langle\begin{array}{lll}
j_{1} & j_{2} & j_{12} \\
j_{3} & j_{4} & j_{23}
\end{array}\right\rangle^{\prime}=q^{\frac{j_{i}}{2}}\left\langle\begin{array}{lll}
j_{1} & j_{2} & j_{12} \\
j_{3} & j_{4} & j_{23}
\end{array}\right\rangle^{\prime} \\
& \hat{y}_{1}\left\langle\begin{array}{lll}
j_{1} & j_{2} & j_{12} \\
j_{3} & j_{4} & j_{23}
\end{array}\right\rangle^{\prime}=\left\langle\begin{array}{ccc}
j_{1}+2 & j_{2} & j_{12} \\
j_{3} & j_{4} & j_{23}
\end{array}\right\rangle^{\prime},
\end{aligned}
$$

we can write $(38)$ as an operator which annihilates the quantum invariants of the tetrahedron graph

$$
\begin{aligned}
& \widehat{A}_{1}\left(\bigotimes ; \hat{y}_{1},\left\{\hat{x}_{i}\right\} ; q\right) \\
= & q^{3} \hat{x}_{3}\left(q^{2}-\hat{x}_{1}^{2}\right)\left(\hat{x}_{1} \hat{x}_{4}-\hat{x}_{23}\right)\left(\hat{x}_{4}-\hat{x}_{1} \hat{x}_{23}\right)\left(\hat{x}_{1} \hat{x}_{2}-\hat{x}_{12}\right)\left(\hat{x}_{2}-\hat{x}_{1} \hat{x}_{12}\right) \hat{y}_{1}^{2} \\
& -\left[q^{2} \hat{x}_{4} \hat{x}_{12}\left(1-\hat{x}_{1}^{2}\right)\left(1-q \hat{x}_{1}^{2}\right)\left(1-q^{2} \hat{x}_{1}^{2}\right)\left(\hat{x}_{3}-\hat{x}_{2} \hat{x}_{23}\right)\left(1-q \hat{x}_{3} \hat{x}_{2} \hat{x}_{23}\right)\right. \\
& +q^{3} \hat{x}_{3}\left(1-\hat{x}_{1}^{2}\right)\left(\hat{x}_{23}-q \hat{x}_{1} \hat{x}_{4}\right)\left(\hat{x}_{1}-\hat{x}_{4} \hat{x}_{23}\right)\left(\hat{x}_{1}-\hat{x}_{2} \hat{x}_{12}\right)\left(\hat{x}_{2}-q \hat{x}_{1} \hat{x}_{12}\right) \\
& \left.+q \hat{x}_{3}\left(1-q^{2} \hat{x}_{1}^{2}\right)\left(\hat{x}_{4}-\hat{x}_{1} \hat{x}_{23}\right)\left(1-q \hat{x}_{1} \hat{x}_{4} \hat{x}_{23}\right)\left(\hat{x}_{12}-\hat{x}_{1} \hat{x}_{2}\right)\left(1-q \hat{x}_{1} \hat{x}_{2} \hat{x}_{12}\right)\right] \hat{y}_{1} \\
& +\hat{x}_{3}\left(1-q^{4} \hat{x}_{1}^{2}\right)\left(\hat{x}_{1}-\hat{x}_{4} \hat{x}_{23}\right)\left(1-q^{2} \hat{x}_{1} \hat{x}_{4} \hat{x}_{23}\right)\left(\hat{x}_{1}-\hat{x}_{2} \hat{x}_{12}\right)\left(1-q^{2} \hat{x}_{1} \hat{x}_{2} \hat{x}_{12}\right) .
\end{aligned}
$$


As $q \rightarrow 1$, the equation 42 reduces to its classical counterpart (36) up to a factor $\left(1-x_{1}^{2}\right)$. The other five equations easily follows from the symmetry property (27)

$$
\begin{aligned}
\widehat{A}_{2}\left(\bigotimes ; \hat{y}_{2},\left\{\hat{x}_{i}\right\}, q\right) & =\widehat{A}_{1}\left(\bigotimes ; \hat{y}_{1} \rightarrow \hat{y}_{2}, \hat{x}_{2} \leftrightarrow \hat{x}_{1}, \hat{x}_{3} \leftrightarrow \hat{x}_{4}\right), \\
\widehat{A}_{3}\left(\bigotimes ; \hat{y}_{3},\left\{\hat{x}_{i}\right\}, q\right) & =\widehat{A}_{1}\left(\bigotimes ; \hat{y}_{1} \rightarrow \hat{y}_{3}, \hat{x}_{3} \leftrightarrow \hat{x}_{1}, \hat{x}_{23} \leftrightarrow \hat{x}_{12}\right), \\
\widehat{A}_{4}\left(\bigotimes ; \hat{y}_{4},\left\{\hat{x}_{i}\right\}, q\right) & =\widehat{A}_{1}\left(\bigotimes ; \hat{y}_{1} \rightarrow \hat{y}_{4}, \hat{x}_{3} \leftrightarrow \hat{x}_{2}, \hat{x}_{1} \leftrightarrow \hat{x}_{4}\right), \\
\widehat{A}_{12}\left(\bigotimes ; \hat{y}_{12},\left\{\hat{x}_{i}\right\}, q\right) & =\widehat{A}_{1}\left(\bigotimes ; \hat{y}_{1} \rightarrow \hat{y}_{12}, \hat{x}_{3} \leftrightarrow \hat{x}_{23}, \hat{x}_{1} \leftrightarrow \hat{x}_{12}\right), \\
\widehat{A}_{23}\left(\bigotimes ; \hat{y}_{23},\left\{\hat{x}_{i}\right\}, q\right) & =\widehat{A}_{1}\left(\bigotimes ; \hat{y}_{1} \rightarrow \hat{y}_{23}, \hat{x}_{3} \leftrightarrow \hat{x}_{12}, \hat{x}_{1} \leftrightarrow \hat{x}_{23}\right) .
\end{aligned}
$$

In fact, these recurrence relations can be considered as the actions of line operators on the $S$-duality wall of $\mathcal{N}=2 S U(2)$ supersymmetric gauge theories with $N_{f}=4$ [9].

\section{Discussions}

In this letter, we have proposed extensions of two conjectures: (i) the generalized volume conjecture for trivalent graphs (ii) the AJ conjecture for trivalent graphs. Particularly, our elaborate exercise for the theta and tetrahedron graphs provides a strong evidence for both the conjectures. To confirm that both the conjectures are true in these cases, it requires to derive the expressions of the classical character variety by gluing equations of ideal tetrahedra triangulations of the complement. Even if the conjectures are true, the configurations of character varieties obtained in this paper provide only local information. The further investigation has to be undertaken to study their global structure.

Although we have dealt with only the theta and tetrahedron graphs in this paper, one can also consider general planar trivalent graphs as well as knotted trivalent graphs [28]. However, we do not expect that the large color asymptotics and recursion relations with respect to only one color would provide character varieties of any trivalent graphs. In fact, in the context of multi-component links, recursion relations involving multi-colors have to be taken into account in order to determine the character variety of the complement in general. Therefore, we rather expect that there are $E$ recursion relations involving multi-colors

$$
\widehat{A}_{i}\left(\Gamma ;\left\{\hat{x}_{j}\right\},\left\{\hat{y}_{k}\right\} ; q\right) J_{n_{1}, \cdots, n_{E}}(\Gamma ; q)=0, \quad(i=1, \cdots, E) .
$$

whose classical limits determine the character variety of a trivalent graph.

In the case of knots, both the conjectures are extended for the HOMFLY polynomials colored by symmetric representations [1, 11, 14]. To the contrary, in the case of $U_{q}\left(\mathfrak{s l}_{N}\right)$ quantum invariants of a trivalent graph, the fusion rule does not allow to increase just one of the colors for representations of $U_{q}\left(\mathfrak{s l}_{N}\right)$. Hence, there is no recursion relation with respect to only one of the colors for higher rank quantum invariants of trivalent graphs. This is the reason why an expression for quantum $6 j$-symbols of $U_{q}\left(\mathfrak{s l}_{N}\right)$ [26] for a simplest class of multiplicity-free representations cannot be written as an Askey-Wilson polynomial. Consequently, the recursion relations for $U_{q}\left(\mathfrak{s l}_{N}\right)$ quantum invariants of trivalent graphs are much more complicated involving multiple colors and general Young tableaux. The feasibility is beyond the scope of our current techniques.

It is well known that the quantum $6 j$-symbols expressed in terms of the Askey-Wilson polynomials ${ }_{4} \varphi_{3}$ exhibit remarkably rich symmetries and dualities. Translating this property in the context of the $3 \mathrm{~d} / 3 \mathrm{~d}$ correspondence, the dualities turn out to be $3 \mathrm{~d}$ mirror symmetries. According to [8], there are 40 descriptions in terms of $\mathcal{N}=2 U(1)$ gauge theory and 32 descriptions in terms of $\mathcal{N}=2 S U(2)$ gauge theory [27] for the 3d gauge theory corresponding to $6 j$-symbols. The interpretation of these relationships in terms of Stokes phenomena of holomorphic blocks [3] is currently under investigation. 


\section{Acknowledgement}

S.N. would like to thank T. Dimofte, S. Gukov and R. van der Veen for valuable discussions. The work of S.N. is partially supported by the ERC Advanced Grant no. 246974, "Supersymmetry: a window to non-perturbative physics".

\section{References}

[1] Aganagic, M. and Vafa, C.: Large N Duality, Mirror Symmetry, and a Q-deformed Apolynomial for Knots. arXiv:1204.4709.

[2] Askey, R., Wilson, J.A.: A set of orthogonal polynomials that generalize the Racah coefficients or 6-j symbols. SIAM J. Math. Anal. 10 (1979), no. 51008.

[3] Beem, C., Dimofte, T., Pasquetti, S.: Holomorphic Blocks in Three Dimensions. arXiv:1211.1986.

[4] Constantino, F.: 6j-symbols, hyperbolic structures and the Volume Conjecture. Geometry \& Topology 11 (2007). arXiv:math/0611399.

[5] Constantino, F.: Integrality of Kauffman brackets of trivalent graphs. arXiv:0908.0542.

[6] Constantino, F., Murakami, J.: On SL(2, C) quantum $6 j$-symbol and its relation to the hyperbolic volume. arXiv:1005.4277.

[7] Constantino, F., Guéritaud, J., Van der Veen, R.: On the volume conjecture for polyhedra. arXiv:1403.2347.

[8] Dimofte, T., and Gaiotto, D.: An E7 Surprise. JHEP 1210 (2012) 129, arXiv:1209.1404.

[9] Dimofte, T., Gaiotto, D. and van der Veen, R.: RG Domain Walls and Hybrid Triangulations, arXiv:1304.6721

[10] Dimofte, T. and van der Veen, R.: A Spectral Perspective on Neumann-Zagier, arXiv:1403.5215

[11] Fuji, H., Gukov, S. and Sulkowski, P.: Super-A-polynomial for knots and BPS states. Nucl.Phys. B867 (2013) 506-546, arXiv:1205.1515.

[12] Garoufalidis, S.: On the characteristic and deformation varieties of a knot. Geometry \&Topology Monographs. 7 (2004) 291-309, arXiv:math/0306230.

[13] Garoufalidis, S., Le, T.: The colored Jones function is $q$-holonomic. Geometry \&Topology. 9 (2004) 1253-1293, arXiv:math/0309214.

[14] Garoufalidis, S.: The colored HOMFLY polynomial is q-holonomic. arXiv:1211.6388.

[15] Goldman, W.M.: The complex-symplectic geometry of SL (2, C)-characters over surfaces, arXiv:math/0304307

[16] Gukov, S.: Three-dimensional quantum gravity, Chern-Simons theory, and the A polynomial. Commun.Math.Phys. 255 (2005) 577-627, arXiv:hep-th/0306165

[17] Hitchin, N.J.: The self-duality equations on a Riemann surface. Proc. London Math. Soc. 55 (1987), no. 3 59-126.

[18] Kachurik, I.I., Klimyk, A.U.: On Racah coefficients of the quantum algebra $U_{q}\left(s u_{2}\right)$. Journal of Physics A: Mathematical and General 23 (1990), no. 132717.

[19] Kashaev, R.: The Hyperbolic volume of knots from quantum dilogarithm. Lett.Math.Phys. 39 (1997) 269-275.

[20] Kirillov, A. N.: Clebsch-Gordan quantum coefficients. Journal of Soviet Mathematics 53.3 (1991): 264-276. 
[21] Kirillov, A.N., Reshetikhin, N.Yu.: Representations of the algebra $U_{q}(\operatorname{sl}(2))$, q-orthogonal Polynomials and Invariants of Links. New Developments in the Theory of Knots, ed. T. Kohno, p.202 (World Scientific, Singapore 1989)

[22] Masbaum, G., Vogel, P.: 3-valent graphs and the kauffman bracket. Pacific Journal of Mathematics 164 (1994), no. 2 361-381.

[23] Murakami, H., Murakami, J.: The colored Jones polynomials and the simplicial volume of a knot. ACTA MATHEMATICA 186 (2001), no. 1 85-104, arXiv:math/9905075.

[24] Murakami, J., Ushijima, A.: A volume formula for hyperbolic tetrahedra in terms of edge lengths. arXiv:math/0402087.

[25] Murakami, J., Yano, M.: On the volume of a hyperbolic and spherical tetrahedron. Comm. Anal. Geom. 13 (2005), 379-400.

[26] Nawata, S., Ramadevi, P., Zodinmawia : Multiplicity-free quantum $6 j$-symbols for $U_{q}\left(\mathfrak{s l}_{N}\right)$. Lett.Math.Phys. 103 (2013) 1389-1398. arXiv:1302.5143.

[27] Teschner, J. and Vartanov, G.S.: $6 j$ symbols for the modular double, quantum hyperbolic geometry, and supersymmetric gauge theories. arXiv:1202.4698.

[28] Van der Veen, R.: The volume conjecture for augmented knotted trivalent graphs. Algebraic \& Geometric Topology (2009), 9, p. 691-722. arXiv:0805.0094.

[29] Varshalovich, D.A., Moskalev, A.N., Khersonskii, V.K.: Quantum Theory Of Angular Momentum. World Scientific (1988). 